\title{
EL CLÚSTER DE SALUD EN MEDELLÍN, VENTAJA COMPETITIVA ALTERNATIVA PARA LA CIUDAD
}

\section{RESUMEN}

$\boldsymbol{E}$ l presente artículo es una explicación del porqué el Clúster de salud en la ciudad de Medellín, tras su consolidación, se puede convertir en una ventaja competitiva alternativa de alto valor agregado para la ciudad, con miras a la exportación de servicios médicos y odontológicos, caracterizado por el vanguardismo en investigación y desarrollo en procedimientos médicos especializados.

El alto nivel desarrollado en Salud, unido a una adecuada consolidación de los sectores educativos, industriales,

comerciales (turismo) y de servicios (transporte), tanto de la ciudad como de otras zonas del departamento de Antioquia, complementan el desarrollo de la iniciativa con el fin de generar factores diferenciales

de competitividad en materia social y económica.

En la actualidad, la estrategia Clúster de la ciudad de Medellín y Antioquia, le apuesta a cinco sectores económicos que se enuncian en el artículo; es por ello que los autores argumentan cómo el Clúster de Salud presenta actualmente el mayor potencial competitivo en comparación con los otros cuatro.

\section{Palabras clave}

Clúster

Salud

Servicios
Jorge Andrés Marulanda Montoya* Geovanny Correa Calle** Luis Fernando Mejía Mejía****

\section{Introducción}

En el municipio de Medellín, desde el año 2006, se inició el trabajo de consolidación del Clúster de salud, denominado "servicios de medicina y odontología", con la finalidad de desarrollar este importante renglón para la competitividad de la ciudad, basa-dos en los grandes logros que han tenido diversas instituciones locales en el desarrollo de procedimientos vanguardistas a nivel mundial en servicios médicos especializados.

* Consultor Independiente, Docente Universitario Postgrado. Administrador de Empresas Ceipa. Especialista de Mercadeo Universidad Católica de Oriente. Candidato a Maestría Gestión de Organizaciones EANUQAC (Medellín).

** Jefe logística Sumicol - Corona. Docente Universitario Postgrado. Administrador Empresas Universidad EAFIT. Especialista Six Sigma (Cinturon negro), Candidato a Maestría Gestión de Organizaciones EANUQAC (Medellín).

*** Analista Senior Banco de Occidente. Docente Universitario Postgrado. Psicólogo Universidad San Buenaventura. Especialista Gerencia de Mercadeo Universidad EAFIT. Candidato a Maestría Gestión de Organizaciones EAN - UQAC (Medellín). 
Es por ello que se ha propuesto explicar cómo dicho clúster, tras su consolidación integral en sí misma y en otros sectores se vislumbra como una fuente de ventaja competitiva de Medellín de talla internacional.

En ese orden de ideas, se propone una descripción de diversos factores que inciden en el clúster de salud, iniciando desde la definición y contextualización del concepto Clúster, para luego desarrollar un ejercicio comparativo con otros países y ciudades del mundo, que muestra experiencias de éxito en este en esta estrategia, para luego llegar al entorno local, planteando los aspectos propios de Medellín, los cuales son el objeto de estudio del articulo. Paso seguido, se plantean una serie de propuestas de consolidación del clúster en la ciudad, la cadena de valor propuesta y las conclusiones generales.

\section{DEFINIENDO EL CONCEPTO DE CLÚSTER}

En un contexto altamente competitivo, en el cual se enmarca la globalización dentro de la economía mundial, se encuentra que más que empresas, Fajnzylber establece que son los sistemas productivos, esquemas institucionales y organizaciones sociales, los que compiten en los mercados internacionales (Citado en CIE, 2005).

\section{ABstraCt}

This paper provides an explanation on why the Health cluster in Medellin may turn into an alternative competitive advantage of great added value for the city. This aims at the possibility of exporting medical services characterized by a vanguard spirit in research and the development of specialized medical procedures.

The high health level reached, together with the proper consolidation of the educational, industrial, commercial, and service sectors of the city and other places in the region of Antioquia, complement the development of the initiative aiming at generating differential issues in the field of socio- economic competitiveness. Currently, the strategic cluster of Medellin city and the region of Antioquial wants to develop five economic sectors that are described above. For this reason, the authors show how the health cluster has the major competitive potential compared with the other four sectors.

\section{KEY WORDS}

Cluster

Health

Services 
Es entonces que la empresa, como nodo fundamental de la competitividad y la innovación, está integrada a una red compuesta por los proveedores, el sistema financiero, el sistema educativo, tecnológico, energético, de transportes, telecomunicaciones, así como por la infraestructura y la calidad del sector público y por las relaciones en la propia empresa. Los rezagos en dichos ámbitos afectan su competitividad, por lo que construir sistemas integrados exige avances simultáneos en la red de contactos que define a la organización; tal como lo afirma Porter (1990), cuando establece que una firma no llegará a ser competitiva por si sola sin apoyo de un ambiente de oferentes y servicios de producción eficientes y sin la presencia de los competidores.

De acuerdo con el Profesor Michael Porter de la Universidad de Harvard, un clúster es un grupo de compañías y asociaciones interconectadas aparentemente divergentes, las cuales están geográficamente cerca, se desempeñan en un sector industrial similar, y están unidas por una serie de características comunes y complementarias, estableciendo que la competitividad de dichas industrias está basada en cuatro factores: firmas competititivas con visión estratégica, exigente demanda doméstica e internacional, alta capacidad de industrias relacionadas y un buen ambiente de instituciones especificas de apoyo, (Porter, citado en Altenburg, Hillebrand, Meyer-Stamer, 1998) para promover el crecimiento económico y social de una región, resaltando la participación de todos los agentes relevantes de educación, empresa y estado.

En economías con clústers consolidados, la creación de riqueza está por encima del promedio regional y tienden a exportar un alto porcentaje de su producción. Pueden incluir desde una región o ciudad, hasta una red de países vecinos. Dentro de estos, se pueden encontrar firmas de todos los tamaños, compañías de productos finales o servicios, proveedores de insumos especializados, componentes, maquinaria, y servicios, instituciones financieras, firmas en industrias relacionadas, instituciones de educación, centros de investigación, instituciones gubernamentales y en general, toda organización que de alguna manera influya en el desempeño del clúster. 


\section{Servicios de SALUd eVidencias Globales}

Es importante mencionar que el mercado potencial de los servicios de salud son muy alentadores, muestra de ello es que en el año 2007, 750 mil estadounidenses viajaron fuera de su país a hacerse algún tratamiento médico, y se espera que en el 2010 esta cifra aumente hasta 6 millones, en donde el 39\% de potenciales clientes estimaron como probable viajar al exterior a realizarse algún procedimiento quirúrgico, si esto implica un ahorro significativo en el costo que ocasionaría hacerse el mismo tratamiento en Estados Unidos. Además, se evidencia que en el mundo el sector creció el $3 \%$ en 2006 , alcanzando un tamaño de mercado de US\$41,6 miles de millones, donde Latinoamérica registró el mayor crecimiento a nivel mundial. ${ }^{1}$

Ante la demanda de Servicios de Salud internacionalmente, son varios los países que han institucionalizado una industria Medica de exportación ("Medical Tourism industry"), aprovechando sus ventajas competitivas para el fortalecimiento de esta actividad. Algunos de los más populares destinos de medicina turística que se destacan por la alta calidad a costos asequibles con referencia a Estados Unidos y países desarrollados son: Argentina, Brasil, Costa Rica, Cuba, India, Malasia, México, Panamá, Filipinas, Sudáfrica, Tailandia y Turquía.

En el año 2007, más de 2.9 millones de pacientes visitaron a Tailandia, Singapur, India, Malasia y Filipinas generando ganancias de US\$3.4 billones y contabilizando el 17.6 por ciento del mercado global. ${ }^{2}$

A su vez, India ha incrementado su turismo básico en un $27 \%$ debido al turismo médico. Se espera que para el año 2012 se tengan entre U\$1.1 a 2.2 billones en ingresos por dicho concepto. (passi publications, 755)

Como ejemplo se puede hacer referencia a Tailandia, que en el año 2002 atendió alrededor de 600.000 turistas y tuvo beneficios económicos por US\$503 millones, ${ }^{3}$ dichos datos comparados con la actualidad muestran un millón de pacientes y ganancias de US\$1.1 billones, apoyados en una fuerte diversificación, incursionando en otros campos médicos alternativos como la homeopatía, el unani y la ayurvéda (Chanda 2006;36) ${ }^{4}$.

\footnotetext{
1 Estudio de Deloitte "Medical Tourism, Consumer in Search of Value" Euromonitor International: Global Sector Briefing. Abril 2008

${ }^{2}$ O Velasco, Noel - Asia News Network 10/2008, http://www.asianewsnet.net

${ }^{3}$ Homeopatía: catalogada por la OMS como CAM (complementary and alternative medicine) la cual se caracterizado por el uso de remedios carentes de ingredientes químicamente activos.

${ }^{4}$ Unani: Medicina de origen Musulmán se basa en principios metafísicos y cosmogonícas. Ayuryéda: Medicina basada en las tradiciones védicas que significa ciencia de la vida y la cual busca integrar el cuerpo, mente y espíritu.
} 
En un contexto regional, se encuentra que a nivel latinoamericano, Cuba fue el pionero, iniciando su estrategia en la década de 1980, la cual consistió en desarrollar su población en diversas especialidades médicas fuera de las fronteras, además de capacitar estudiantes que venían del exterior y brindar atención y tratamiento a pacientes extranjeros. Para lograrlo crearon una compañía que denominaron Servimed, la cual busco mercadear y comercializar paquetes de salud con operadores turísticos. Actualmente este rubro representa U\$25 millones al año para la economía de la Isla ${ }^{1}$.
Las referencias anteriores a nivel internacional y latinoamericano, permiten apreciar como el mercado del turismo medico ha crecido de manera exponencial en cada uno de los países que lo han involucrado como una de sus políticas de competitividad nacional. Sin embargo, no se podría afirmar que los casos antes expuestos indiquen clúster altamente consolidados, más bien se refieren al desarrollo del sector de servicios médicos específicos. Por ello se han presentado las anteriores referencias con el fin de mostrar lo atractivo del sector para el desarrollo de un clúster local en salud, que integre las competencias y las características de las que no se tiene evidencias de los casos de éxito internacionales.

\section{LA EXPERIENCIA COLOMBIANA EN LA CREACIÓN DE CLÚSTER DE SALUD}

El gobierno Colombiano, mediantedelDepartamentodePlaneación Nacional (DNP), inicia en el año 2004 la construcción de la Agenda Interna para la Competitividad y Productividad, de allí surge la estrategia competitiva del sector Salud con base en Clústers Regionales, los cuales se basan en el desarrollo de posibilidades de exportación de servicios de Salud, que se encuentran actualmente fortalecidos por la alta calidad médica, la adecuada infraestructura hospitalaria, los desarrollos investigativos y el vanguardismo en el tema de trasplantes a nivel internacional. Ya que estos servicios potencializan una oportunidad de generar divisas y una alternativa de desarrollo a los sistemas de salud locales.

Actualmente en el país, las iniciativas de Clústers de Salud se han dado en la ciudad de Bogotá con el programa Salud Capital, por medio de un proyecto conjunto entre la Cámara de

${ }^{1}$ The George Washington University - Economic data. http://www1.american.edu/ted/medical-tourism.htm 
Comercio de Bogotá, la Alcaldía Mayor y proexport. Otro elemento referencial es la Ciudad de Cali, a través del programa Valle de la Salud, liderado por la Universidad Javeriana, el cual estimula el desarrollo de Capacidades competitivas del sector. En el departamento de Santander por su lado, se está gestionando el proyecto Salud Santander. Y por último se encuentra que en la ciudad de Medellín se desarrolla la estrategia Clúster, liderada por la Cámara de Comercio y la Alcaldía de la Ciudad. También en otras regiones se vislumbran esfuerzos de manera independiente, como es el caso del Eje Cafetero, Barranquilla y Cartagena, los cuales se encuentran en etapa formulación.

Según la Agenda interna del sector Salud (DNP), para el año 2019, la visión es lograr altos estándares de calidad, investigación y desarrollo nacionales e internacionales que faciliten la competitividad en el sector salud a todos los niveles ${ }^{1}$, y planteando a su vez siete estrategias para lograr llegar a cumplir los estándares exigidos a nivel global, siendo estas las siguientes ${ }^{2}$ :

- Estabilidad jurídica y normativa. (Marcos legales claros y estables).
- Incremento de la productividad del sector (mediante actualización, desarrollo tecnológico y capacitación humana).

- Fortalecimiento Comercial del Sector Salud. (Equidad en costos, investigación y estrategias de marketing).

- Gestión de la información del Sector Salud. (construcción de un sistema de información interinstitucional).

- Gestión de los recursos. (estatales y privados).

- Diagnóstico y adecuación. (Infraestructura, tecnología y acreditación).

- Clúster de Salud (Consolidaciones Regionales).

Actualmente existen siete departamentos que le apuestan al desarrollo y fortalecimiento del sector salud con base a sus ventajas comparativas y especialidades, estos son: Antioquia, Atlántico, Región Bogotá -Cundinamarca-, Valle del Cauca Santander, Caldas y Risaralda (DNP).

\footnotetext{
DNP - Agenda interna para la productividad y la competitividad - Documento sectorial Salud, Agenda interna Sectorial. Pg.24
}

2 DNP - Documento Sectorial Salud, Pgs 24-27. 


\section{UNA VOCACIÓN PIONERA DEL SECTOR SALUD DE ANTIOQUIA EN EL MUNDO ${ }^{1}$}

En la década de los 90 , ante una oferta de servicios médicos de baja calidad en Centroamérica y el Caribe, en relación a los altos costos en Estados Unidos, la ciudad de Medellín empezó a recibir un gran número de pacientes procedentes de las Antillas Holandesas. Fue entonces que entidades como el Hospital Universitario San Vicente de Paul, inició la atención a pacientes del exterior por sus áreas de trasplantes, dentro de los cuales se encuentra el primer trasplante doble de pulmón, siendo el primero a nivel latinoamericano y reconocido a nivel mundial.

Ante estas nuevas oportunidades de mercado, diversas entidades del sector salud de la ciudad se unen en el año 2000 para formar el programa Salud sin Fronteras, conformado por la Clínica Cardiovascular Santa María, El Hospital Universitario San Vicente de Paul, El Hospital Pablo Tobón Uribe,
La Clínica Medellín, La Clínica las Américas, La Clínica el Rosario, La Clínica las Vegas y Emergencia Médica Integral (EMI) como apoyo logístico. La alianza de estas ocho instituciones permitió la disponibilidad de 1.074 médicos generales y especialistas, 1.309 enfermeras auxiliares y profesionales, 91 unidades de cuidados intensivos, 30 unidades de diagnostico, 73 quirófanos y 1.653 camas $^{2}$ para prestar servicios en 15 ramas de la medicina.

Haciendo una correlación con la información anterior, se evidencia que entre los años 2000 y 2003, la ciudad de Medellín atendió alrededor de 3.500 pacientes extranjeros, donde es importante destacar la evolución de US $\$ 950.000$ exportados en salud en el año 2000 a una extraordinaria cifra cercana de US $\$ 3$ millones en el año 2004. Veamos algunos datos de Salud sin Fronteras ${ }^{3}$. (Ver cuadro 1 y 2 ).

\footnotetext{
1 DNP - Documento Sectorial Salud, Pgs 16-18

2 DNP - Documento Sectorial Salud, Pg 17.

${ }^{3}$ DNP - Documento Sectorial Salud, Pg 17.
} 
CUADRO 1

Cuadro comparativo ingresos sector salud año 2000 a 2004

\begin{tabular}{|c|c|r|}
\hline Año & No. de pacientes & \multicolumn{1}{c|}{ Ingresos en US\$ } \\
\hline 2000 & 505 & 950.000 \\
\hline 2001 & 460 & 1.500 .000 \\
\hline 2002 & 636 & 1.700 .000 \\
\hline 2003 & 900 & 2.000 .000 \\
\hline $2004(\mathrm{e})$ & 1000 & 3.000 .000 \\
\hline
\end{tabular}

\section{CUADRO 2}

\begin{tabular}{|l|l|}
\hline \multicolumn{2}{|c|}{ Sitios de origen de los pacientes } \\
\hline $2000-2002$ & 822 personas de Estados Unidos \\
\hline $2002-2003$ & 138 de Centromaérica \\
\hline $2002-2004$ & 161 del Caribe \\
\hline $2002-2005$ & 480 de otros paises \\
\hline
\end{tabular}

Fuente: Departamento Nacional de Planeación

Las actividades del sector salud no solo se remiten a los procesos netamente médicos, sino que también se enfocan a la realización de visitas de observación a otros países para establecer contactos, promoción, interacción de buenas prácticas, conocimiento del mercado y la recolección de información de la oferta de hospitales y clínicas a todo nivel, con lo cual se retroalimenta la experiencia local con la internacional y se enriquece la curva de aprendizaje de las entidades del clúster. 


\section{Razones para la consolidación deL CLÚSTER DE SALUd EN MEDELLÍN}

Son muchas las razones para que Medellín convierta su Clúster de Salud en un modelo ejemplar de desarrollo económico y social de talla mundial. Como referencia se cuenta con la reconocida gestión en materia de salud a nivel internacional que viene gestionando varias entidades de la ciudad desde hace varios años, muestra de ello es la
Asociación Nacional de Trasplantados y la Corporación para el Fomento de Trasplantes. Dichas Instituciones realizan trabajos complementarios al grupo de trasplantes de la Universidad de Antioquia y el Hospital Universitario San Vicente de Paul, el cual, con treinta años de funcionamiento, ha realizado el mayor volumen de trasplantes en el país (Ver Cuadro 3).

CUADRO 3

Número de transplantes por regional a 2007

\begin{tabular}{|l|c|l|l|l|}
\hline Regional & Renal & Hepático & Cardiaco & Totales \\
\hline 1. Bogotá & 284 & 66 & 18 & 368 \\
\hline 2. Antioquia & 245 & 86 & 27 & 358 \\
\hline 3. Valle & 91 & 41 & 18 & 150 \\
\hline 4. Santander & 36 & 0 & 6 & 42 \\
\hline 5. Atlántico & 4 & 0 & 0 & 4 \\
\hline Totales & 660 & 193 & 69 & 922 \\
\hline
\end{tabular}

Fuente: Ministerio de la Protección Social 2007. República de Colombia.

El equipo mencionado anteriormente, realizó en 1973 el primer trasplante de riñón exitoso en Colombia, cuando la técnica aún se estaba perfeccionando en el mundo. Luego, en 1979, efectuó el primer trasplante de hígado en Latinoamérica. Así mismo, en 1985, realizaron el primero de corazón del país en cooperación con la Clínica Cardiovascular. Paso seguido, fue el primero de páncreas de Suramérica en ese mismo año, más otros de córnea, medula ósea, corazón-pulmón, y combinaciones como los de riñón-páncreas e hígado-riñón, hasta culminar con el primero de laringe y de tráquea del mundo en 2008, gracias a una técnica quirúrgica desarrollada exclusivamente por colombianos.

Según los intereses de diferentes entidades estatales en el desarrollo de Clústers: la Cámara de Comercio de Medellín y la Alcaldía de Medellín, manifiestan que los empresarios 
latinoamericanos, perciben a la ciudad como un centro atractivo para el desarrollo de actividades de la salud. ${ }^{1}$ Ver gráfico 1.

\section{GRÁFICO 1}

Atractividad en Actividades de Salud de Medellín

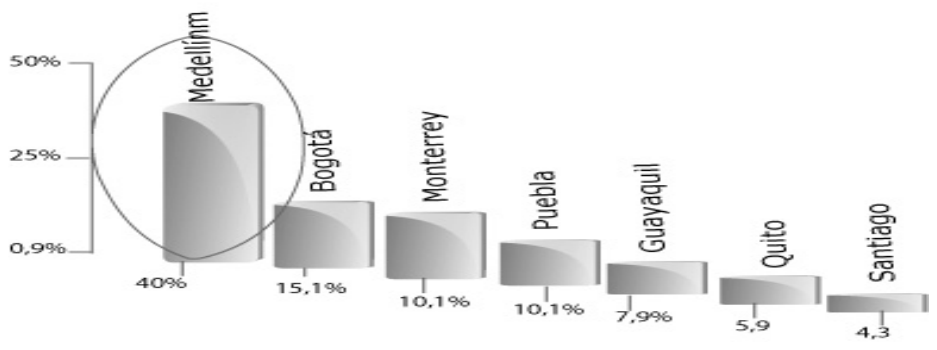

Fuente: Amércia Economía Intelligence. 2008.

Estas percepciones y los ejercicios hasta ahora desarrollados en materia de trasplantes, hacen de Medellín una ciudad con un futuro de grandes proporciones de crecimiento en cuanto a la prestación de servicios médicos especializados; pero más allá de la obtención de ingresos económicos, es traducir esta oportunidad en un verdadero salto al desempeño de nivel superior, que le permita a la ciudad salirse de los esquemas tradicionales de generación de desarrollo y competitividad de corto plazo.

En dicha línea, la ciudad inicio con la estrategia Clúster en el año 2006, centrándose en cinco áreas que son: Clúster de Energía Eléctrica, Clúster de Construcción, Clúster textil / Confección, diseño y moda, Clúster de Turismo de Negocios, ferias y convenciones y por último el Clúster de servicios de medicina y odontología, donde los dos últimos se caracterizan por su liderazgo en la construcción de redes y la generación de nuevos negocios, con el fin de mejorar la competitividad de ciudad².

El clúster de salud de Medellín, es la concentración geográfica de empresas e instituciones especializadas y complementarias en la actividad de medicina, odontología, educación e investigación, producción y/o comercialización de insumos hospitalarios, dispositivos, tecnología biomédica, telemedicina, producción y/o distribución de medicamentos, desarrollo de software científico y conocimiento; las cuales interactúan entre sí, creando un clima de negocios en el que todos pueden mejorar su desempeño, competitividad y rentabilidad.

\footnotetext{
1 2o Foro Internacional de Calidad. Ministerio de la Protección Social Oferta de valor por regiones Cámara de Comercio de Medellín para Antioquia Alcaldía de Medellín. Clusters de Competitividad. Bogotá, diciembre 01-02 de 2008.

${ }^{2}$ Documento Comunidad Clúster Número 5, Cámara de Comercio de Medellín, Alcaldía de Medellín, 2009.
} 
Dicho Clúster, brinda amplios beneficios a las empresas que lo componen y puede convertirse en un factor determinante de la competitividad de la economía de Medellín, los cuales incluyen ${ }^{1}$ :

- Incremento de los niveles de experticia. Provee a las empresas un mayor grado de apropiación de su cadena de valor, y permite cooperación y aprendizaje potencial entre las mismas.

- Aumenta la capacidad de las empresas de inducir habilidades complementarias.

- Aumento de la productividad de las empresas en el Clúster y posibilidad de desarrollo de nuevos negocios.

- Fortalecimiento social y otros vínculos informales, bases para la creación de nuevas ideas y nuevos negocios y para el incremento de la eficiencia en investigación y desarrollo.

- Mejoramiento de los flujos de información.
- Facilitamiento del desarrollo de una infraestructura de servicios profesionales, legales, financieros, entre otros.

- Fortalecimiento de la coordinación y transacciones entre empresas, permitiendo la difusión de mejores prácticas, lo que a su vez estimula y facilita la innovación, y promueve la creación y el desarrollo de instituciones creadoras y proveedoras de conocimiento.

- Fortalecimiento de la atracción y permanencia de capitales extranjeros, lo que a su vez puede posibilitar el fortalecimiento de capacidades industriales mediante la trasferencia tecnológica, lo cual permite un proceso de upgrade en la base de capital y crea por ende una economía más prospera y sofisticada (Lall, 2003).

- Posibilitan la inserción de la base productiva de la región a las cadenas mundiales de valor (Lall, 2003).

1 Englansd's Regional Development Agencies. 


\section{FACTORES CLAVE DE MEDELLÍN PARA EL} CLÚSTER DE SALUD

a salud representa en el Valle de Aburrá un conglomerado empresarial de 3.277 empresas con activos totales por USD $\$ 2.064$ millones, según cifras de la Unidad de Investigaciones Económicas de la Cámara de Comercio. De estas, un 88.59 por ciento son microempresas, el 8.42 por ciento pequeñas, el 2.01 por ciento medianas y solo en 0.98 por ciento son grandes. Por esta razón, el nuevo clúster le apunta al fortalecimiento empresarial y a la creación de nuevas empresas, ya que posee un talento humano competitivo y altamente cualificado, que aprende y emprende, que es dinámico y sensible a las necesidades humanas. De igual modo se cuenta con un adecuado desarrollo de infraestructura y recursos logísticos para el desarrollo de la actividad, por lo que la ciudad se proyecta en la consolidación de negocios estratégicos y mejoramiento de la calidad de vida de sus habitantes. (Fuente, Cultura E, Programa Líder de la Alcaldía de Medellín para el desarrollo empresarial).

El Valle de Aburrá ${ }^{1}$ posee grandes ventajas competitivas entre las que se destacan los bajos costos administrativos y la alta cobertura en la distribución de los excedentes a través de proyectos sociales y productivos, además de servicios públicos de la mejor calidad y cobertura en aspectos como: agua, electricidad, educación y salud; de la misma manera se cuenta con mano de obra abundante con más de 3'305.211 habitantes en capacidad laboral (Dane 2005), una dinámica cada vez mayor en la investigación, ligadas al sistema productivo, y una excelente infraestructura financiera y grandes centros dedicados al comercio de talla mundial ${ }^{2,3}$.

Con la ayuda de la $\mathrm{ACl}$ (Agencia internacional de la cooperación) y La Cámara de Comercio, La ciudad de Medellín está desarrollando un proyecto estratégico en promoción de la ciudad, concentrada en dos campos: En primer lugar se encuentra la creación de mayores ventajas competitivas para la actividad emprendedora, que respondan a las exigencias de los mercados extranjeros y en segunda instancia está la promoción de grupos industriales que se pueden considerar estratégicos, debido a su importancia en la economía por factores como la generación de empleo y la dinámica en la internacionalización de los mercados.

\footnotetext{
1 Valle de Aburrá un territorio de diez municipios que de manera dinámica se integran formando una gran ciudad. Los municipios que la conforman son Barbosa, Bello, Caldas, Copacabana, Envigado, la Estrella, Girardota, Itagüí, Medellín, Sabaneta.

2 Estado de la Actividad Financiera, Económica y Social Periodo Enero 01 a Diciembre 31 de 2008. Municipio de Medellín.

3 Medellín Adelante y Sin Reversa: "Why Medellín?, documento de la Alcaldía de Medellín 2006.
} 
Con lo anteriormente mencionado, con la consolidación del Clúster de Salud, la ciudad de Medellín se proyecta a erigirse como un centro de desarrollo de proyectos de salud en: investigación, oferta de servicios médicos, infraestructura hospitalaria, trasplantes, cirugías estéticas, entre otros; que propician fuentes de desarrollo económico para sectores complementarios como el turismo, el transporte y la actividad comercial; generando soportes estratégicos desde la academia, el estado, la estabilidad jurídica y normativa, los sectores privados dedicados al transporte aéreo y terrestre y el turismo complementario; soportado en sistemas de información interinstitucional para compartir conocimiento y mejores prácticas con las mejores experiencias del mundo en salud.

\section{8. ¿QUÉ ACCIONES SE DEBEN PRIORIZAR PARA LA CONSOLIDACIÓN DEL CLÚSTER?}

El lector desprevenido, podría comenzar haciendo una lista de requerimientos de orden tecnológico, procedimientos, formación en profesionales e inversión en infraestructura para actividades de la salud; sin embargo se considera pertinente definir lo que éste proyecto requiere para su consolidación, prioritariamente en aspectos como:

\subsection{Declaración de voluntad política:} es quizás la lección más difícil que se ha tratado de aprender a lo largo de los años, no considerada sólo como la voluntad de los políticos, ni de quienes participan de manera más evidente en la vida política de la nación. Es considerar a los líderes en todos los ámbitos de la vida (los grupos profesionales, el sector privado, los sindicatos, las instituciones religiosas y otros grupos de la sociedad civil, por mencionar algunos) y evaluar alternativas de cómo se pueden movilizar para ayudar a mejorar la calidad de vida y el desarrollo de la región.

8.2 Trabajo Integrado: entre los diferentes entes públicos: empresas privadas, las instituciones universitarias, el gobierno, los gremios relacionados y las instituciones extranjeras aliadas para el desarrollo de proyectos exitosos asociados con actividades de la salud.

8.3 Alineación a planes de desarrollo: dentro de la Agenda de Productividad y competitividad de Antioquia, se debe incluir el clúster de salud como una línea estratégica clave en el desarrollo del Valle de Aburrá, definiendo acciones y 
planes de inversión y presupuestos orientados a su desarrollo. Es fundamental mencionar en este punto, la relevancia que toma para la propuesta, la integración de acciones alineadas en los diversos planes de distinto orden, tanto nacional, departamental, como municipal.

\subsection{Estrategia de posicionamiento} de la ciudad: es evidente el gran avance que se ha hecho en este sentido, donde la imagen de Medellín en el ámbito nacional e internacional se ha tornado muy favorable, ello como una ciudad con potencial de desarrollo sustentado en los índices de desempeño y la gestión administrativa, la calidad de la gente y las ventajas comparativas y competitivas en áreas relacionadas que le aportarán el Clúster de la Salud un alto valor agregado.

\subsection{Inclusión del sectorsaludenelplan} de desarrollo de Medellín: significa esto, que el plan de desarrollo para Medellín contenga además de los sectores tradicionales, un plan especial para el sector Salud, con líneas estratégicas, objetivos claros (generales y específicos), con presupuesto y líneas de inversión que permitan elevar el nivel de participación y desempeño de las actividades en salud, como foco de generación de desarrollo y riqueza para la ciudad, teniendo en cuenta acciones en el mismo sentido de todos los municipios de área metropolitana.
Se hace necesario entonces, socializar este tema con los actores claves de la Ciudad y el Valle de Aburrá, además de presentar argumentos básicos para orientar la atención al desarrollo estratégico del Clúster de la Salud en Medellín. Sin embargo, para su desarrollo sostenible se requiere de un trabajo deliberado de los actores, en busca de enfocar la oferta de productos y servicios básicos a un nivel superior de atención de manera inteligente, con alto valor agregado, que potencialice a Medellín como una ciudad de la salud, partiendo de las grandes fortalezas que posee la Región en torno a su talento humano, y asimismo, las debilidades en infraestructura y transporte que hacen que la relación precio/costo logístico sea considerablemente alto; de tal forma que éste esfuerzo completo se debe enfocar en la estrategia de atracción y retención de la inversión tanto local como extranjera, soportados con planes coherentes entre sí.

En ese orden de ideas, los siguientes aspectos enunciados se consideran de gran importancia, puesto que son factores cruciales para la consolidación del clúster:

- Fortalecimiento y consolidación del Clúster de la salud de Antioquia, en cuyo principal foco de acción es la ciudad de Medellín, contando con una estructura funcional definida y un liderazgo fundamentado en procesos de innovación y mejora. En ese orden de ideas se considera fundamental la convocatoria a todas las Instituciones 
de salud públicas y privadas que fomenten sinergias en conocimiento, procesos e infraestructura, con el fin de que el desarrollo del clúster alcance niveles de desempeño de clase mundial con oferta de valor exportable.

- Desarrollo de un plan estratégico que responda en esencia a como comprender y enfrentar las necesidades del mercado, especialmente en el sector de servicios especializados de la salud. Este plan debe ser la carta de navegación hacia la consecución de objetivos establecidos conjuntamente con los actores directos, teniendo en cuenta que la participación de otros sectores indirectos como el transporte, la hotelería, entre otros, deben estar alineados con dicha estrategia.

- Definición del portafolio de productos y servicios de acuerdo con criterios de calidad y cumplimiento de normas y legislación existente tanto a nivel nacional como internacional. Es importante señalar, que el portafolio de servicios de la salud en su fase inicial, deberá estar subordinado a la capacidad científica actual en materia de: infraestructura, oferta de profesionales, productos y servicios complementarios y tecnología disponible. Es importante mencionar que se debe generar una fuerte orientación a la innovación y desarrollo de los aspectos mencionados, que procure por atraer inversión de capital público, privado y extranjero, como factor clave para el verdadero desarrollo del clúster ${ }^{1}$.

- Creación de un ente de soporte en investigación y desarrollo, cuyas funciones sean el fortalecimiento $y$ gestión del Clúster, ello en materia de enfermedades, gestión tecnología para los procedimientos, la biotecnología, ortopedia, investigación y diseño de procesos, generación de servicios complementarios, ajuste a la normatividad en salud, entre otros. Dicho ente tiene como finalidad hacer las veces de gerencia del proyecto.

- Definición de estándares de calidad de los productos, servicios y procedimientos, ello por la vía de la certificación y acreditación ante entes privados, gubernamentales, nacionales e internacionales.

- Diseño y definición de los diferentes perfiles de conocimiento de los profesionales en salud. En un trabajo conjunto con las instituciones universitarias, las instituciones acreditadoras, el Estado e institutos privados en salud. Desarrollando programas de formación coherentes con las necesidades del clúster.

- Definición de la capacidad exportadora acerca de los servicios y productos que se está en capacidad de ofertar,

\footnotetext{
2o Foro Internacional de Calidad Ministerio de la Protección Social Oferta de valor por regiones Cámara de Comercio de Medellín para Antioquia Alcaldía de Medellín Clusters de Competitividad Bogotá, diciembre 01-02 de 2008.
} 
orientado a las ventajas comparativas y competitivas de la ciudad frente a la oferta internacional.

Además de los puntos enunciados anteriormente, se considera de gran importancia la definición de una estrategia de marketing de salud muy fuerte (Factor que se encuentra en un pobre desarrollo actualmente), enlazadas a un trabajo en posicionamiento de marca fuerte, alto valor agregado por medio de la innovación y factores de diferenciación global (Estrategia PAD, Universidad EAN), con el fin de ser altamente competitivos en el mercado.

Así mismo, como elemento propositivo, se presenta un esquema de cadena de valor, que permita optimizar los esfuerzos y tienda por el desarrollo de la ventaja competitiva deseada con la consolidación del Clúster de Salud, por medio de la integración efectiva de los principales factores que intervienen en el esquema. (Ver gráfico 2)

GRÁFICO 2

Cadena de valor de Clúster, relación de factores clave

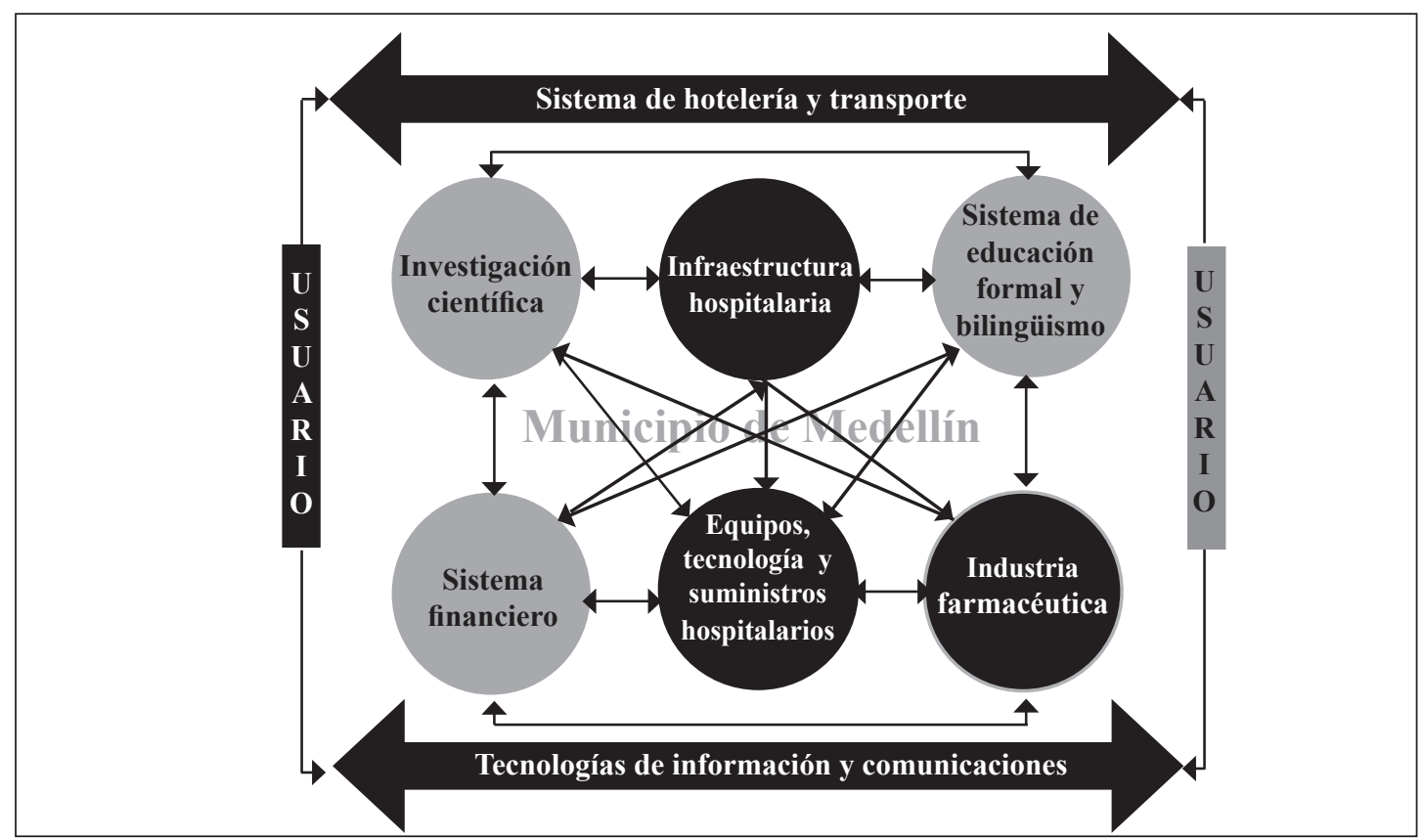

Fuente: propia

Como complemento al anterior esquema de cadena de valor, se presenta en el gráfico 3 la interrelación de actores necesarios para la operatividad efectiva del esquema propuesto, evidenciando la integración institucional necesaria para el Clúster de Salud. 
GRÁFICO 3

Actores del Clúster

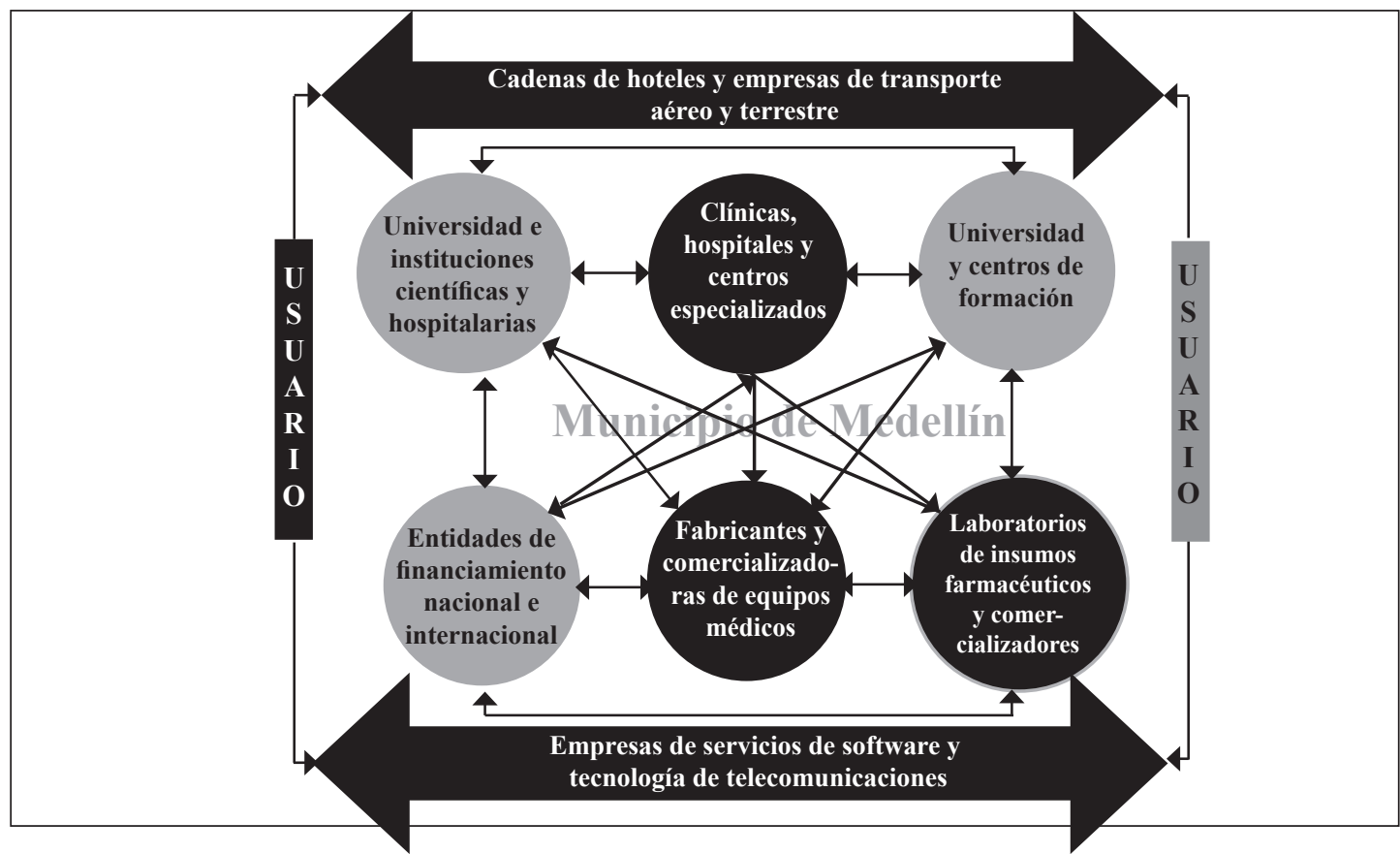

Fuente: propia

\section{Integración de Clústers como estrategia PARA EL INCREMENTO DE LA COMPETITIVIDAD Y LA GENERACIÓN DE VALOR AGREGADO}

Es importante anotar que en la actualidad, El desarrollo de la estrategia Clúster en la ciudad de Medellín de desarrollade formaautónoma, siendo esta opción considerada como inapropiada en el planteamiento de servicios de salud, donde se considera de gran relevancia la efectiva integración entre sectores, en nuestro caso especifico: el turismo y el transporte.

\section{Integración Clúster -Turismo}

Dicho clúster se convierte en un elemento transversal en el desarrollo y crecimiento del Clúster Salud, del cual depende la generación efectiva de medios logísticos y promocionales de la ciudad. 
Veámos algunas características de este sector en la ciudad:

Actualmente por sus importantes transformaciones en seguridad e infraestructura, la ciudad de Medellín se presenta como uno de los destinos turísticos más importantes del país. Según Cotelco el porcentaje de ocupación Hotelera de Antioquia en el año 2008 fue del $51.1 \%$ ocupando el séptimo lugar en comparación al 1ro que fue ocupado por Bogotá con el $64.3 \%$.

Si nos remitimos a la agenda interna para la productividad y competitividad del DNP y específicamente el documento sectorial de Turismo, la visión para Medellín y su área Metropolitana solo está orientada a negocios. Sin embargo en el mismo documento en las apuestas regionales para el sector turismo se amplía el concepto a la biodiversidad, los negocios y los eventos.

Según Cotelco entre 2004 y 2008 la ciudad ha desarrollado 13 proyectos hoteleros incrementando su capacidad de camas en 11.959. Sin embargo actualmente no existe hotelería con características de alojamiento Médico Post-operatorio (Definiendo el término como el proceso de recuperación sin intervención hospitalaria y médica directa).

En dicho caso, la industria hotelera debe tener presente para este hospedaje Post-Operatorio las siguientes facilidades como:
- Accesos amplios, como rampas, puertas, ascensores, corredores.

- Baños amplios que permita ingresar una silla de ruedas, caminador, etc.

- Duchas tipo manguera, soportes de seguridad y accesorios para colgar.

- Flexibilidad en la preparación de los alimentos con base en las indicaciones medico-nutricionales que trae el huésped post-operado.

- Apoyo logístico de ambulancia o coches especiales.

- Capacitación especializada a su personal en los factores de atención y colaboración. (Muchas personas desconocen como movilizar un paciente operado).

- A solicitud de los huéspedes, posibilitarles mediante un convenio previo con instituciones del sector salud personal de enfermería profesional o auxiliar.

Por otra parte, se tiene que las posibilidades del Clúster de turismo, no solo se limita a la cadena hotelera, sino también a renglones económicos fuertes como el ecoturismo, Ferias y convenciones, Moda y comercio (centros comerciales).

Como estrategia, se considera que la integración de este clúster debe ser orientada por un ente de promoción 
exclusivo, que garantice la promoción de servicios tanto en el sector salud como de manejo del los tiempos nulos de los pacientes pre y pos-operados.

\section{Integración Clúster -Transporte}

En la actualidad, Medellín cuenta con un $14 \%$ de la capacidad instalada para servicios especiales de transporte terrestre, sin embargo se debe hacer un gran esfuerzo para generar medios adecuados en atención y movilización de pacientes extranjeros, ya que dicho porcentaje no se orienta a personas en condiciones medicas y odontológicas, que permitan asegurar un alto confort y cuidado especial. Para ello se deben generar practicas de investigación y desarrollo, que brinden todos los medios técnicos para asegurar una plena satisfacción de los pacientes.

Para ello se hace necesario generar nuevos servicios orientados a transportar pacientes y por defecto acoger a adultos mayores o personas con limitación, por eso se deben tener presente aspectos como acceso al vehículo, bodegaje de silla de ruedas, muletas, caminador, etc.

Con las facultades de ingeniería y diseño de la ciudad, en las universidades como Eafit, la Escuela de
Ingenieros de Antioquia y la Pontificia Bolivariana, se podrá lograr alianzas para el desarrollo ingenioso de puertas, rampas de acceso, montacargas portátil, grúas de elevación portátil, accesorios de soporte y demás necesidades que se puedan ir determinando a medida que los pacientes utilicen los servicios. Estos diseños pueden tener grandes posibilidades de exportación.

En cuanto a transporte aéreo, el clúster de salud proporcionaría el incremento de pasajeros durante todo el año y no de manera estacional. Las aerolíneas que puedan proporcionarles a estos clientes post-operados algunas características de atención a bordo, diferentes a las del común del mercado lograrán ser la primera opción de selección. Algunas características a tener en cuenta son:

- Preferencia y facilidad en el Check in.

- Manejo del equipaje.

- Ayuda con su equipaje de mano.

- Almohadas y cobijas extras.

- Menú alimenticio especial.

Es importante mencionar que en todos los medios de transporte requeridos (aéreo y terrestre), se debe hacer un proceso de formación y capacitación en servicio especial a los clientes, además del manejo de varios idiomas y conocimiento profundo de las características del clúster. 


\section{Conclusión}

Se encuentra que en la actualidad el desarrollo local está fundamentado en sectores que poseen dificultades en la generación valor agregado y diversificación de cara al progreso económico por sus característica tradicionales, es por ello que se toma la consolidación del clúster de salud como una importante alternativa que orienta una actividad productiva con alta especialización, diferenciación, investigación, desarrollo e innovación, con miras a impactar un mercado global con importantes ventajas competitivas.
Podemos concluir que la consolidación del clúster debe ser prioritaria para la ciudad, con la inclusión de otros sectores complementarios como el turismo y el transporte que brinden a los clientes gran confiabilidad en los servicios prestados, que permita la asociación de Medellín, a condiciones adecuadas de seguridad, amabilidad, respeto por la calidad de vida y una población altamente globalizada de cara al mundo. 


\section{(11. Bibliografía}

Alcaldía de Medellín. Estado de la Actividad Financiera, Económica y Social. Periodo Enero 01 a Diciembre 31 de 2008.

Clúster de Turismo de Negocios, www.medellin.gov.co, www.acimedellin.org.

Comisión Regional de Competitividad. www.proantioquia.gov.co

Cámara de Comercio de Medellín. Documento: 2o. Foro Internacional de Calidad Ministerio de la Protección Social Oferta de valor por regiones. Antioquia Alcaldía de Medellín Clusters de Competitividad, Bogotá, diciembre 01-02 de 2008.

DNP - Departamento Nacional de Planeación: Agenda Interna para la Productividad y la Competitividad, documento Sectorial Salud.

La Ciudad de los Trasplantes, Sol Astrid Giraldo. Revista Dinero.

Medellín en Cifras, Alcaldía de Medellín. Según los resultados del estudio de PROBIDAD, encuesta 2.006, Programa de Eficiencia y Rendición de Cuentas.

Medical Tourism Represents a $\$ 2.1$ billion business, study shows. Tuesday, September 23, 2008; By Steve Twedt, Pittsburgh Post-Gazette.

Plan de Desarrollo Antioquia 2008 - 2011, www.govant.gov.co.

Políticas y programas de salud en América Latina. Problemas y propuestas. Documento de la CEPAL, Irma Arriagada, Verónica Aranda y Francisca Miranda. División de Desarrollo Social. Santiago de Chile, diciembre de 2005.

Servicios de Medicina y Odontología, un nuevo clúster para Medellín. Max Banner Ads. Abril de 2002.

The Rise of Medical Tourism Q\&A with: Tarun Khanna Published: December 17, 2007. Author. Martha Lagace. 
Turismo de salud un sector de talla mundial. Euromonitor international: Global Sector Briefing. April 2008.

Universidad Javeriana: Modelo Teórico y Análisis Empírico para la exportación en Servicios de Salud. Centro de proyectos para el desarrollo. Documento de trabajo ASS /DT 012B- 04; Autores: Fernando Ruiz Gómez, Enrique Peñaloza Quintero y Liz Garavito Beltrán. Bogotá, Colombia.

¿WHY MEDELLíN? Medellín Adelante y Sin Reversa. Alcaldía De Medellín, 2006.

www.acimedellin.org

www.minprotecciónsocial.gov.co

www.cta.org.co 